\title{
Heterosis for Cane, Juice Yield and its Component Traits in Sweet Sorghum
}

\author{
S. R. Aru ${ }^{1 *}$, D.V. Kusalkar ${ }^{2}$, U.S. Dalvi ${ }^{3}$, M.S. Shinde ${ }^{3}$, \\ A.S. Totre ${ }^{1 *}$, A.S. Jadhav ${ }^{3}$ and V.S. Wani ${ }^{4}$ \\ ${ }^{1}$ Department of Agricultural Botany MPKV, Rahuri, (MS), India \\ ${ }^{2}$ Zonal Agril. Research Station, Igatpuri, MPKV, Rahuri (MS), India \\ ${ }^{3}$ Sorghum Improvement Project MPKV, Rahuri (MS), India \\ ${ }^{4}$ Department of Statistics, MPKV, Rahuri (MS), India \\ *Corresponding author
}

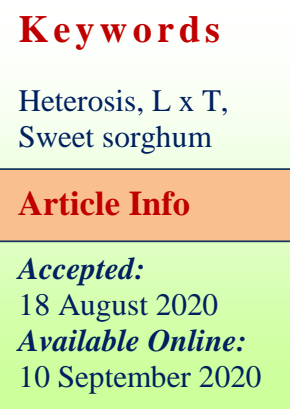

\section{Keywords}

Heterosis, L x T,

Article Info

Accepted:

18 August 2020

10 September 2020

\section{A B S T R A C T}

The experiment was undertaken on Line $\mathrm{x}$ Tester analysis for, juice yield and its component traits in crosses of A and R lines of sweet sorghum at All India Coordinated Sorghum Improvement Project, M.P.K.V., Rahuri, during the year 201718 with objectives to study the heterosis of parents and hybrids. The CMS lines (females), ten testers (males) and their thirty $\mathrm{F}_{1}$ 's hybrids were studied by using $\mathrm{L}$ $\mathrm{x} T$ design. Observations were recorded on fourteen characters viz., Days to $50 \%$ flowering, Days to physiological maturity, Internode / Plant, Plant height $(\mathrm{cm})$, Stem girth $(\mathrm{cm})$, Total biomass yield (t/ha), Cane weight $(\mathrm{t} / \mathrm{ha})$, Cane harvest index (\%), Juice yield (lit/ha), Brix (\%), Reducing sugar (\%), Non-reducing sugar $(\%)$, Total sugar (\%), Computed ethanol yield (lit/ha). Among the fourteen characters studied, majority of the characters exhibited mid-parents as well as better parent heterosis along with standard heterosis in desirable direction in most of the hybrids, indicating the predominant role of non- fixable inter-allelic interactions and over dominance in the expression of heterosis in respect of all these traits.

\section{Introduction}

Sorghum is recognized as one of the best crops for biomass energy feedstock. The sugar and starch in sweet sorghum could be fermented to ethanol and liquid cellulose could be gasified to methane. The concept of high energy sorghum is now gaining forward. The recent national bio-fuel policy of government of India state that an indicated target of $20 \%$ percent ethanol blending with petrol viewed largely a measure of environmental sustainability and reduced the dependence on fossil fuels. The traditional route of producing ethanol as product of sugar industry will not meet this huge demand. Therefore, as demand for production of fuel ethanol, through renewable sources increase 
to unprecedented levels, feed stock for ethanol production will become more diverse. High biomass sweet sorghum would be a best option owing to its wider adoption. Therefore, sweet sorghum being a water use efficient non-invasive crop having under adaptability will meet the requirement of upcoming ethanol firms that has not only significant impact on sustaining the environment but also on livelihood opportunities at small farmer in semi-arid tropics.

Heterosis or hybrid vigour is the increased or decreased vigour growth, fitness or yield of a hybrid over the parental values, resulting from the crossing of genetically divers genotype. Heterosis has positive association with specific combining ability (sca) variance. For the development of effective heterosis breeding programme in sweet sorghum, one needs to have information about genetic architecture and estimated prepotency of parents in hybrid combinations.

\section{Materials and Methods}

The experimental material for the present study comprised of three male sterile lines, ten restorers, their resulting 30 hybrids and one hybrid check Phule Vasundhara. During rabi 2017- 18 three male sterile lines and ten restores were sown at Sorghum Improvement Project, M.P.K.V., Rahuri and these lines and testers were crossed in Line $\mathrm{x}$ Tester design to produce 30 possible hybrids. The experiment was conducted during kharif 2018 by using thirteen parents, their 30 hybrids along with one standard check Phule Vasundhara at Sorghum Improvement Project, M.P.K.V., Rahuri. The observations were recorded on fourteen characters viz., Days to $50 \%$ flowering, Days to physiological maturity, Internode / Plant, Plant height $(\mathrm{cm})$, Stem girth $(\mathrm{cm})$, Total biomass yield $(\mathrm{t} / \mathrm{ha})$, Cane weight (t/ha), Cane harvest index (\%), Juice yield (lit/ha), Brix (\%), Reducing sugar (\%),
Non-reducing sugar (\%), Total sugar (\%), Ethanol yield (lit/ha). In the present investigation heterosis has been estimated over mid parent (Average / Relative heterosis) and better parent (heterobeltiosis) as per Fonesca and Patterson (1968).

\section{Results and Discussion}

Heterosis, a fundamental tool, used for the improvement of crops in the form of $F_{1}$ and $F_{2}$ populations by improving the various yield contributing characters. The magnitude of heterosis was reported among the crosses, demonstrating potential of hybrid combinations of thirteen diverse parents for various traits enhancement in the present research. It is observed that, the mean squares due to lines, testers as well as lines vs tester interaction and hybrids are found significant for all the characters under studies, except the magnitude of variance in lines and testers is found non-significant for Internodes per plant, in lines it is non significant for total sugar and also for magnitude of variance in lines and lines vs. testers found non significant for brix (Table 1). Mean performance of parents for cane yield, juice yield per hectares and its contributing characters in sweet sorghum are presented in Table 2. Higher values are desirable for all traits under study except for days to $50 \%$ flowering, days to maturity and reducing sugar per cent for which lower values are preferred.

The mean performance of hybrids for different traits studied were compared with the corresponding mid parent (MP), better parent (BP) and standard check hybrids (Phule Vasundhara) and the differences are being expressed as per cent heterosis for cane, juice yield, its components traits. In sweet sorghum, positive heterosis was desirable for all the characters studied except days to $50 \%$ flowering, days to maturity and reducing sugar where negative heterosis is desirable. 
Character wise results of average heterosis (H1) heterobeltiosis (H2) and standard heterosis (H3) observed in the 30 crosses (Table 3).

Earliness is a desirable character that helps to develop early maturity variety. The standard heterosis for days to $50 \%$ flowering ranged from -11.24 to -26.59 per cent over check Phule Vasundhara (Table 3). The crosses ICMS-479A x RSSV-498, ICMS-479A $x$ RSSV-512, CMS-1409A x RSSV-498 (-26.59 $\%)$ exhibits highest negative standard heterosis followed by cross 1409A x RSSV453 (-26.22), ICMS-479A x RSSV-503 ($26.22 \%)$ and 1409A x RSSV-512 (-26.22\%). All 30 hybrids showed significant negative standard heterosis over check Phule Vasundhara. These results are in line with the earlier results of Sandeep et al., (2009), Vinaykumar (2009), Bahadure et al., (2015) and Kumar et al., (2016). Days to maturity is often closely correlated with days to flowering, although genetic differences in the period or duration required for flowering to maturity exists. Heterosis over standard check (Phule Vasundhara) was negative in direction for most of the hybrids indicating earliness of hybrids (Table 3 ). The crosses ICMS-479A x RSSV-509, CMS-1409A x RSSV-498 (18.93\%) and CMS-1409A x RSSV-453 ($18.67 \%$ ) showed highest negative standard heterosis followed by cross ICMS-479A x RSSV-542 (-18.41) and ICMS-479A $x$ RSSV-542(-18.41\%). All 30, crosses recorded highly significant negative standard heterosis over check, Phule Vasundhara. Internode per plant in sorghum is one of the important character, Out of 30 hybrids, 19 hybrids recorded significant average heterosis in positive direction. The heterosis over better parent ranged from -2.78 per cent (CMS- 185 $\mathrm{x}$ RSSV-542) to 27.27 per cent (CMS-185 X RSSV-509) and 4 hybrids recorded significant heterobeltiosis for Internode per plant. Out of 30 crosses, none of the cross exhibited positive and significant standard heterosis for this trait.

Plant height is an important character in sweet sorghum. All the 30 crosses, show highest significant positive average heterosis in desirable direction for plant height. Heterobeltiosis ranged from -0.56 (CMS-185 $\mathrm{x}$ RSSV-503) to 20.69 per cent (CMS-185 x RSSV-509) (Table 3). As the check Phule Vasundhara is tall in growth habit the standard heterosis for plant height ranged from -0.08 to 4.53 per cent over check Phule Vasundhara (Table 3). Four crosses showed standard heterosis in desirable direction. Maximum positive heterosis was found in crosses ICMS-479 x RSSV-260 (4.53\%) followed by CMS-185 x RSSV-509 (2.77\%) and CMS-1409 x RSSV-512 (2.51\%), while maximum negative standard heterosis was observed in ICMS-479 x RSSV-386 ($30.84 \%$ ). Similar result recorded by Agarwal and Shrotria (2005), Sandeep et al., (2009), Vinaykumar (2009). Out of 30 hybrids, 22 hybrids recorded significant average heterosis and heterobeltosis in positive direction for stem girth. Only 4 cross combination were exhibited positive and significant standard heterosis for stem girth. Maximum positive heterosis was found in crosses ICMS-479 $\mathrm{x}$ RSSV-260 (9.62\%) followed by CMS-185 x RSSV-260 (9.06\%) and CMS-1409 x RSSV$503(8.27 \%)$. The heterosis over better parent was ranged from -1.40 per cent (CMS-185 x RSSV-313) to 43.23 per cent (ICMS-479 X RSSV-260) for total biomass yield. A total of 26 hybrids recorded positive significant heterosis over better parent. The cross ICMS479 X RSSV-260 (43.23\%) recorded highest positive significant heterosis over better parent. The highest significant standard heterosis recorded in the cross, ICMS-479 X RSSV-260 (10.25\%), followed by cross CMS-185 X RSSV-260 (5.15\%) and CMS1409 X RSSV-260 (5.14\%). The similar results were earlier reported by Sandeep et al., 
(2009), Vinaykumar (2009), Pfeiffer et al., (2010), Tariq et al., (2014), Bahadure et al., (2015) and Kumar et al., (2016) (Table 3).

Cane weight of a genotype serves as an indicator of juice yield as it is an important character contributing to juice yield in sweet sorghum. Average heterosis for cane weight ranged from 16 to 107.31 per cent. Heterobeltiosis ranged from -1.94 (CMS-185 x RSSV-313) to 68.04 per cent (ICMS-479 x RSSV-260) (Table 3). Out of 30 crosses, 24 crosses show highest significant positive heterobeltiosis in desirable direction for can weight. Standard heterosis over check hybrid Phule Vasundhara ranged from -3.91 per cent (CMS-1409 x RSSV-386) to 14.30 per cent (ICMS-479 X RSSV-260). Eight crosses showed standard heterosis in desirable direction. The highest significant standard heterosis recorded in the cross, ICMS-479 X RSSV-260 (14.30\%), followed by cross CMS-185 X RSSV-260 (7.22\%) and CMS1409 X RSSV-260 (7.13\%). The similar results were earlier reported by Sandeep et al., (2009), Vinaykumar (2009), Pfeiffer et al., (2010), Tariq et al., (2014), Bahadure et al., (2015) and Kumar et al., (2016) (Table 3). Can harvest index is an important parameter in sweet sorghum. Average heterosis for cane weight ranged from 6.67 to 28.89 per cent. Heterobeltiosis ranged from -0.55 (CMS185A x RSSV-313) to 17.38 per cent (ICMS479A x RSSV-260). Standard heterosis over check hybrid Phule Vasundhara ranged from 1.14 per cent (CMS-1409A x RSSV-386) to 3.66 per cent (ICMS-479A x RSSV-260). Six crosses showed standard heterosis in desirable direction. The magnitude of heterosis over mid-parent for juice yield ranged from -1.98 per cent (CMS-185 X RSSV-313) to 93.25 per cent (ICMS-479 X RSSV-260). A total of 19 hybrids recorded significant positive heterosis over mid parent. The range in heterobeltiosis varied from -0.34 per cent (CMS-185 x RSSV-498) to 72.60 per cent
(ICMS-479 X RSSV-260). A total of 16 hybrids recorded significant positive heterosis over better parent. The range of heterosis over standard checks, Phule Vasundhara was from 2.04 per cent (ICMS-479 X RSSV-509) to 9.41 per cent (ICMS-479 X RSSV-260). Among 30 hybrids, 2 hybrids recorded positive significant heterosis over Phule Vasundhara. The highest standard heterosis in desirable direction was recorded in the cross ICMS-479 X RSSV-260 (9.41\%), followed by cross CMS-1409 x RSSV-260 (4.57\%) Similar result recorded by Vinaykumar (2009), Pfeiffer et al., (2010), Sidramappa et al., (2012), Tariq et al., (2014), and Kumar et al., (2016) (Table 3). Average heterosis for percentage of brix ranged from -0.95 to 21.90 per cent. Maximum significant positive average heterosis recorded in CMS-1409 X RSSV-509 (21.90\%) followed by ICMS-479 x RSSV-509 (17.31\%). The heterobeltiosis ranged from -1.89 to 20.75 per cent. 10 crosses recorded significant positive heterobeltiosis. The range of heterosis over standard checks, Phule Vasundhara was from -3.64 per cent (CMS-1409 X RSSV-542) to 16.36 per cent (CMS-1409 X RSSV-509). The cross CMS-1409 X RSSV-509 (16.36\%) exhibited highest significant heterosis over better parent followed by ICMS-479 X RSSV-509 (10.91\%) and CMS-1409 X RSSV-498.). Similar finding reported by Meshram et al., (2005), Rajashekhar (2007), Vinaykumar (2009) and Sidramappa et al., (2012). Average heterosis for percentage of reducing sugar ranged from -2.33 to 64.71 per cent. Maximum significant negative average heterosis recorded in ICMS-479 x RSSV-386 $(27.45 \%)$. The heterobeltiosis ranged from 2.33 to 156.67 per cent. None of the cross recorded significant negative heterobeltiosis. Standard heterosis for this trait ranged from 1.43 to 10 per cent. Out of 30 crosses 21 crosses exhibited negative and significant standard heterosis for this trait. 
Table.1 Analysis of variance for combining ability and estimates of gca and sca variances in sweet sorghum

\begin{tabular}{|c|c|c|c|c|c|c|c|c|}
\hline Sources & DF & $\begin{array}{c}\text { Days to } \\
50 \% \\
\text { flowering }\end{array}$ & $\begin{array}{l}\text { Days to } \\
\text { maturity }\end{array}$ & $\begin{array}{c}\text { Internodes/ } \\
\text { Plant }\end{array}$ & $\begin{array}{l}\text { Plant height } \\
\quad(\mathrm{cm})\end{array}$ & $\begin{array}{l}\text { Stem girth } \\
(\mathrm{cm})\end{array}$ & $\begin{array}{c}\text { Total } \\
\text { biomass } \\
\text { yield (t/ha) }\end{array}$ & $\begin{array}{c}\text { Cane } \\
\text { weight } \\
\text { (t/ha) }\end{array}$ \\
\hline Replication & 2 & 0.49 & 0.70 & 2.077 & 20.98 & 0.008 & 0.84 & 0.69 \\
\hline Treatments & 42 & $116.59 * *$ & $112.25 * *$ & $6.84 * *$ & $6944.82 * *$ & $5.53 * *$ & $217.95 * *$ & $217.83 * *$ \\
\hline Parents & 12 & $312.17 * *$ & $300.17 * *$ & $9.47 * *$ & $15115.92 * *$ & $0.77 * *$ & $172.72 * *$ & $171.32 * *$ \\
\hline Line & 2 & $3.11 *$ & $2.78 * *$ & 0.44 & $1013.78 * *$ & $0.19 * *$ & $39.54 * *$ & $38.29 * *$ \\
\hline Testers & 9 & $342.00 * *$ & $326.90 * *$ & 1.55 & $804.45 * *$ & $0.98 * *$ & $34.78 * *$ & $34.79 * *$ \\
\hline Line vs. Tester & 1 & $661.88 * *$ & $654.38 * *$ & $98.80 * *$ & $172123.48 * *$ & 0.005 & $1680.48 * *$ & $1666.13 * *$ \\
\hline Parent vs. hybrid & 1 & $145.22 * *$ & $156.84 * *$ & $36.66 * *$ & $46282.02 * *$ & $57.97 * *$ & $3722.59 * *$ & $3722.98 * *$ \\
\hline Hybrids & 29 & $34.67 * *$ & $32.96 * *$ & $4.73 * *$ & $2207.21 * *$ & $5.70 * *$ & $115.81 * *$ & $116.21 * *$ \\
\hline Error & 84 & 0.814 & 2.62 & 1.04 & 20.59 & 0.01 & 0.40 & 0.45 \\
\hline \multicolumn{9}{|l|}{ Estimates } \\
\hline$\sigma_{\text {gca }}^{2}$ & & $3.7679 * *$ & $3.3884 * *$ & $0.2709 * *$ & $187.4018 * *$ & $0.3357 * *$ & $8.9385 * *$ & $8.9780 * *$ \\
\hline$\sigma_{\text {sca }}^{2}$ & & $6.5094 * *$ & $5.9005^{* *}$ & $0.3168 *$ & $406.3949 * *$ & $1.2207 * *$ & $25.5500 * *$ & $25.6523 * *$ \\
\hline$\sigma_{A}^{2}$ & & 7.5358 & 6.7767 & 0.5417 & 374.8036 & 0.6715 & 17.8769 & 17.9561 \\
\hline$\sigma_{D}^{2}$ & & 6.5094 & 5.9005 & 0.3168 & 406.3949 & 1.2207 & 25.5500 & 25.6523 \\
\hline$\sigma^{2}{ }_{A} / \operatorname{var}_{D}$ & & 1.1577 & 1.1485 & 1.7098 & 0.9223 & 0.5501 & 0.6997 & 0.7000 \\
\hline
\end{tabular}

Note: $*$ Significant at $5 \%$ level of significance, $* *$ Significant at $1 \%$ level of significance 
Table. 1 contd...

\begin{tabular}{|c|c|c|c|c|c|c|c|c|}
\hline Sources & DF & $\begin{array}{c}\text { Cane harvest } \\
\text { index }(\%)\end{array}$ & $\begin{array}{c}\text { Juice yield } \\
\text { (lit/ha) }\end{array}$ & Brix (\%) & $\begin{array}{c}\text { Reducing } \\
\text { Sugar } \\
(\%)\end{array}$ & $\begin{array}{c}\text { Total } \\
\text { sugar } \\
(\%)\end{array}$ & $\begin{array}{c}\text { Non } \\
\text { reducing } \\
\text { sugar }(\%)\end{array}$ & $\begin{array}{l}\text { Ethanol yield } \\
\text { (lit/ha) }\end{array}$ \\
\hline Replication & 2 & 0.02977 & 137105.68 & 0.389 & $0.040 *$ & 0.12 & 0.120 & 1057.49 \\
\hline Treatments & 42 & $71.09173^{* *}$ & $17549920.11^{* *}$ & $4.74 * *$ & $0.49 * *$ & $7.52 * *$ & $6.22 * *$ & $106579.82 * *$ \\
\hline Parents & 12 & $93.59920 * *$ & $5504988.15^{* *}$ & $2.07 * *$ & $0.49 * *$ & $10.29 * *$ & $8.21 * *$ & $36768.10 * *$ \\
\hline Line vs. Tester & 1 & $961.39310 * *$ & $25116399.80 * *$ & 0.12 & $3.33 * *$ & $85.45^{* *}$ & $55.61 * *$ & $375094.53 * *$ \\
\hline Parent vs. hybrid & 1 & 1192.82877 & $60148528.57 * *$ & $18.53 * *$ & $0.94 * *$ & $59.21 * *$ & $46.33 * *$ & $689550.74 * *$ \\
\hline Hybrids & 29 & 23.09770 & $21065112.36^{* *}$ & $5.37 * *$ & $0.47 * *$ & $4.59 * *$ & $4.009 * *$ & $115364.98 * *$ \\
\hline$\sigma_{\text {sca }}^{2}$ & & $5.5310 * *$ & $3339231.72 * *$ & $1.6275 * *$ & $0.1573 * *$ & $0.9199 * *$ & $0.7253^{* *}$ & $16776.0130 * *$ \\
\hline$\sigma_{A}^{2}$ & & 3.6971 & 3345217.2663 & 0.6927 & 0.0387 & 1.3567 & 1.1497 & 24238.0923 \\
\hline
\end{tabular}

Note: $*$ Significant at $5 \%$ level of significance, ${ }^{* *}$ Significant at $1 \%$ level of significance 
Table.2 Mean performance of parents for juice yield and its contributing traits in sweet sorghum

\begin{tabular}{|l|l|c|c|c|c|c|c|c|}
\hline $\begin{array}{l}\text { Sr. } \\
\text { No. }\end{array}$ & $\begin{array}{l}\text { Name of } \\
\text { parents }\end{array}$ & $\begin{array}{c}\text { Days to } \\
\text { Flowering }\end{array}$ & $\begin{array}{c}\text { Days to } \\
\text { maturity }\end{array}$ & $\begin{array}{c}\text { Nodes/ } \\
\text { plant }\end{array}$ & $\begin{array}{c}\text { Plant } \\
\text { height } \\
\text { (cm) }\end{array}$ & $\begin{array}{c}\text { Stem } \\
\text { girth } \\
\text { (cm) }\end{array}$ & $\begin{array}{c}\text { Total } \\
\text { biomass } \\
\text { yield } \\
\text { (t/ha) }\end{array}$ & $\begin{array}{c}\text { Cane } \\
\text { weight } \\
\text { (t/ha) }\end{array}$ \\
\hline & Female (Lines) & $\mathbf{1}$ & $\mathbf{2}$ & $\mathbf{3}$ & $\mathbf{4}$ & $\mathbf{5}$ & $\mathbf{6}$ & $\mathbf{7}$ \\
\hline $\mathbf{1 .}$ & CMS-185A & 65 & 105 & 9 & 230 & 6.2 & 42.6 & 22.1 \\
\hline $\mathbf{2 .}$ & ICMS-479A & 63 & 103 & 8 & 210 & 5.8 & 42.8 & 22.4 \\
\hline $\mathbf{3 .}$ & CMS-1409A & 63 & 105 & 8 & 194 & 5.7 & 49.0 & 28.4 \\
\hline & Mean & 63.67 & 104.33 & 8.33 & 211.56 & 5.9 & 44.82 & 24.3 \\
\hline & Male (Testers) & & & & & & & \\
\hline 4. & RSSV-542 & 71 & 111 & 12 & 385 & 6.2 & 60.3 & 39.7 \\
\hline $\mathbf{5 .}$ & RSSV-260 & 101 & 141 & 14 & 388 & 6.4 & 56.6 & 36.0 \\
\hline 6. & RSSV-509 & 67 & 107 & 11 & 337 & 4.9 & 58.8 & 38.2 \\
\hline 7. & RSSV-386 & 77 & 117 & 12 & 367 & 5.5 & 60.4 & 39.9 \\
\hline $\mathbf{8 .}$ & RSSV-498 & 66 & 106 & 12 & 388 & 5.3 & 56.7 & 36.1 \\
\hline 9. & RSSV-417 & 67 & 109 & 12 & 355 & 5.6 & 59.6 & 39.0 \\
\hline $\mathbf{1 0 .}$ & RSSV-453 & 67 & 108 & 12 & 368 & 6.0 & 59.4 & 38.7 \\
\hline $\mathbf{1 1 .}$ & RSSV-313 & 79 & 121 & 12 & 367 & 6.1 & 67.3 & 46.8 \\
\hline $\mathbf{1 2 .}$ & RSSV-512 & 70 & 111 & 13 & 379 & 6.8 & 65.2 & 44.6 \\
\hline $\mathbf{1 3 .}$ & RSSV-503 & 68 & 110 & 13 & 359 & 6.2 & 59.9 & 39.2 \\
\hline & Mean & 73.3 & 114.1 & 12.3 & 369 & 5.92 & 60.4 & 39.8 \\
\hline & SE \pm & 0.5211 & 0.9478 & 0.588 & 2.599 & 0.0617 & 0.3660 & 0.3841 \\
\hline & CD at 5\% & 1.4650 & 2.6646 & 1.653 & 7.3092 & 0.1734 & 1.0290 & 1.0799 \\
\hline & C.V. & 1.2911 & 1.4829 & 8.275 & 1.2425 & 1.5307 & 0.9727 & 1.4916 \\
\hline
\end{tabular}

Note: * Significant at $5 \%$ level of significance, ${ }^{* *}$ Significant at $1 \%$ level of significance. 
Table. 2 contd..

\begin{tabular}{|c|c|c|c|c|c|c|c|c|}
\hline $\begin{array}{l}\text { Sr. } \\
\text { No. }\end{array}$ & $\begin{array}{l}\text { Name of } \\
\text { parents }\end{array}$ & $\begin{array}{c}\text { Cane } \\
\text { harvest } \\
\text { index } \\
(\%)\end{array}$ & $\begin{array}{c}\text { Juice } \\
\text { yield } \\
\text { (lit/ha) }\end{array}$ & $\begin{array}{l}\text { Brix } \\
(\%)\end{array}$ & $\begin{array}{l}\text { Reducing } \\
\text { sugar } \\
(\%)\end{array}$ & $\begin{array}{c}\text { Total } \\
\text { sugar } \\
(\%)\end{array}$ & $\begin{array}{c}\text { Non } \\
\text { reducing } \\
\text { sugar } \\
(\%)\end{array}$ & $\begin{array}{c}\text { Ethanol } \\
\text { yield } \\
\text { (lit/ha) }\end{array}$ \\
\hline & Female (Lines) & 8 & 9 & 10 & 11 & 12 & 13 & 14 \\
\hline 1. & CMS-185A & 51.94 & 7671 & 17.7 & 1.43 & 6.77 & 5.3 & 276.3 \\
\hline 2. & ICMS-479A & 52.19 & 6740 & 17.0 & 1.06 & 7.03 & 5.9 & 250.7 \\
\hline \multirow[t]{3}{*}{3.} & CMS-1409A & 58.00 & 6574 & 17.3 & 1 & 7.10 & 6.0 & 248 \\
\hline & Mean & 54.04 & 6995 & 17.3 & 1.16 & 6.96 & 5.8 & 258.33 \\
\hline & Male (Testers) & & & & & & & \\
\hline 4. & RSSV-542 & 65.86 & 7823 & 19.3 & 1.8 & 12.5 & 10.7 & 519.0 \\
\hline 5. & RSSV-260 & 63.62 & 8573 & 16.7 & 2.2 & 9.9 & 7.7 & 451.0 \\
\hline 6. & RSSV-509 & 64.99 & 8592 & 17.7 & 1.5 & 10.7 & 9.2 & 488.0 \\
\hline 7. & RSSV-386 & 65.94 & 8139 & 17.0 & 2.3 & 8.9 & 6.5 & 382.0 \\
\hline 8. & RSSV-498 & 63.69 & 8742 & 18.3 & 1.6 & 10.7 & 9.0 & 497.0 \\
\hline 9. & RSSV-417 & 65.45 & 7980 & 17.3 & 2.0 & 11.8 & 9.8 & 502.0 \\
\hline 10. & RSSV-453 & 65.32 & 10260 & 16.3 & 1.8 & 10.3 & 8.4 & 559.0 \\
\hline 11. & RSSV-313 & 69.43 & 8176 & 16.7 & 1.9 & 10.8 & 8.8 & 470.7 \\
\hline 12. & RSSV-512 & 68.43 & 9030 & 17.0 & 1.9 & 10.6 & 8.7 & 507.7 \\
\hline \multirow[t]{5}{*}{13.} & RSSV-503 & 65.56 & 11683 & 18.3 & 1.4 & 8.6 & 7.2 & 534.3 \\
\hline & Mean & 65.83 & 8899.8 & 17.5 & 1.86 & 10.48 & 8.59 & 491.067 \\
\hline & SE \pm & 0.0054 & 156.81 & 0.34 & 0.06 & 0.15 & 0.16 & 11.19 \\
\hline & CD at $5 \%$ & 0.0151 & 441 & 0.97 & 0.17 & 0.43 & 0.47 & 31.48 \\
\hline & C.V. & 0.6264 & 2.83 & 3.32 & 5.68 & 2.48 & 3.26 & 3.49 \\
\hline
\end{tabular}

Note: * Significant at $5 \%$ level of significance, $* *$ Significant at $1 \%$ level of significance. 
Table.3 Heterosis (\%) over mid-parent (MP), better-parent (BP) and standard check (Phule Vasundhara) for different characters in sweet Sorghum

\begin{tabular}{|c|c|c|c|c|c|c|c|c|c|c|}
\hline \multirow{3}{*}{$\begin{array}{l}\text { Sr. } \\
\text { No. }\end{array}$} & \multirow[t]{3}{*}{ Crosses } & \multicolumn{3}{|c|}{ Days to $50 \%$ flowering } & \multirow{2}{*}{\multicolumn{3}{|c|}{ Days to maturity }} & \multicolumn{3}{|c|}{ Internodes/plant } \\
\hline & & \multicolumn{3}{|c|}{1} & & & 2 & \multicolumn{3}{|c|}{3} \\
\hline & & MP (H1) & BP(H2) & Check (H3) & MP (H1) & BP(H2) & Check (H3) & MP (H1) & BP (H2) & Check (H3) \\
\hline 1 & CMS-185A x RSSV-542 & $-2.70 * *$ & 2.06 & $-25.84 * *$ & -1.54 & 1.27 & $-18.41 * *$ & 11.11 & -2.78 & $-16.67 * *$ \\
\hline 2 & CMS-185A x RSSV-260 & $-13.08 * *$ & $11.34 * *$ & $-19.10 * *$ & $-8.67 * *$ & $6.98 * *$ & $-13.81 * *$ & $23.53 * *$ & 2.44 & 0.00 \\
\hline 3 & CMS-185A x RSSV-509 & $8.59 * *$ & $10.82 * *$ & $-19.48 * *$ & $5.49 * *$ & $6.67 * *$ & $-14.07 * *$ & $40.00 * *$ & $27.27 * *$ & 0.00 \\
\hline 4 & CMS-185A x RSSV-386 & $1.89 *$ & $11.34 * *$ & $-19.10 * *$ & 1.35 & $6.98 * *$ & $-13.81 * *$ & $25.00 * *$ & 8.11 & -4.76 \\
\hline 5 & CMS-185A x RSSV-498 & $4.33 * *$ & $5.67 * *$ & $-23.22 * *$ & $2.84 *$ & $3.49 * *$ & $-16.62 * *$ & $22.58 * *$ & 8.57 & -9.52 \\
\hline 6 & CMS-185A x RSSV-417 & $3.80 * *$ & $5.67 * *$ & $-23.22 * *$ & $2.65 *$ & $4.44 * *$ & $-15.86 * *$ & $15.63 *$ & 0.00 & -11.90 \\
\hline 7 & CMS-185A x RSSV-453 & $2.54 *$ & $4.12 * *$ & $-24.34 * *$ & 1.88 & $3.49 * *$ & $-16.62 * *$ & 0.00 & -13.51 & $-23.81 * *$ \\
\hline 8 & CMS-185A x RSSV-313 & -0.46 & $10.82 * *$ & $-19.48 * *$ & 0.00 & $7.62 * *$ & $-13.30 * *$ & 0.00 & -13.51 & $-23.81 * *$ \\
\hline 9 & CMS-185A x RSSV-512 & $7.20 * *$ & $11.34 * *$ & $-19.10 * *$ & $6.01 * *$ & $9.21 * *$ & $-12.02 * *$ & $27.27 * *$ & 7.69 & 0.00 \\
\hline 10 & CMS-185A x RSSV-503 & $12.28 * *$ & $15.46 * *$ & $-16.10 * *$ & $6.36 * *$ & $8.89 * *$ & $-12.28 * *$ & 1.54 & -13.16 & $-21.43 * *$ \\
\hline 11 & ICMS-479A x RSSV-542 & 0.25 & $6.91 * *$ & $-24.72 * *$ & -0.78 & $2.90 *$ & $-18.41 * *$ & 8.20 & -8.33 & $-21.43 * *$ \\
\hline 12 & ICMS-479A x RSSV-260 & $-3.46 * *$ & $26.06 * *$ & $-11.24 * *$ & $-3.14 * *$ & $14.52 * *$ & $-9.21 * *$ & $42.42 * *$ & $14.63 *$ & 11.90 \\
\hline 13 & ICMS-479A x RSSV-509 & $2.05 *$ & $5.85 * *$ & $-25.47 * *$ & 0.32 & 2.26 & $-18.93 * *$ & $41.38 * *$ & $24.24 * *$ & -2.38 \\
\hline 14 & ICMS-479A x RSSV-386 & $2.39 *$ & $13.83 * *$ & $-19.85 * *$ & 0.61 & $7.10 * *$ & $-15.09 * *$ & $22.58 * *$ & 2.70 & -9.52 \\
\hline 15 & ICMS-479A x RSSV-498 & 1.29 & $4.26 * *$ & $-26.59 * *$ & 1.75 & $3.23 *$ & $-18.16 * *$ & $33.33 * *$ & 14.29 & -4.76 \\
\hline 16. & ICMS-479A x RSSV-417 & $5.40 * *$ & $9.04 * *$ & $-23.22 * *$ & $3.46 * *$ & $6.13 * *$ & $-15.86 * *$ & 12.90 & -5.41 & $-16.67 * *$ \\
\hline 17. & ICMS-479A x RSSV-453 & $10.31 * *$ & $13.83 * *$ & $-19.85 * *$ & $6.46 * *$ & $9.03 * *$ & $-13.55 * *$ & 9.68 & -8.11 & $-19.05 * *$ \\
\hline 18. & ICMS-479A x RSSV-313 & 0.47 & $13.83 * *$ & $-19.85 * *$ & 0.45 & $9.03 * *$ & $-13.55 * *$ & 9.68 & -8.11 & $-19.05 * *$ \\
\hline 19. & ICMS-479A x RSSV-512 & -1.26 & $4.26 * *$ & $-26.59 * *$ & -0.62 & $3.23 *$ & $-18.16 * *$ & 12.50 & -7.69 & $-14.29 *$ \\
\hline 20. & ICMS-479A x RSSV-503 & 0.25 & $4.79 * *$ & $-26.22 * *$ & 0.63 & $3.87 * *$ & $-17.65 * *$ & $20.63 * *$ & 0.00 & -9.52 \\
\hline 21. & CMS-1409A x RSSV-542 & -1.24 & $4.74 * *$ & $-25.47 * *$ & -0.31 & $2.54 *$ & $-17.39 * *$ & $21.31 * *$ & 2.78 & -11.90 \\
\hline 22. & CMS-1409A x RSSV-260 & $-11.97 * *$ & $14.21 * *$ & $-18.73 * *$ & $-7.59 * *$ & $8.25 * *$ & $-12.79 * *$ & $27.27 * *$ & 2.44 & 0.00 \\
\hline 23. & CMS-1409A x RSSV-509 & $2.55 *$ & $5.79 * *$ & $-24.72 * *$ & 2.04 & $3.17 *$ & $-16.88 * *$ & $37.93 * *$ & $21.21 * *$ & -4.76 \\
\hline 24. & CMS-1409A x RSSV-386 & $-5.24 * *$ & $4.74 * *$ & $-25.47 * *$ & $-3.76 * *$ & 1.59 & $-18.16 * *$ & $25.81 * *$ & 5.41 & -7.14 \\
\hline 25. & CMS-1409A x RSSV-498 & 0.77 & $3.16 * *$ & $-26.59 * *$ & 0.00 & 0.63 & $-18.93 * *$ & $26.67 * *$ & 8.57 & -9.52 \\
\hline 26. & CMS-1409A x RSSV-417 & $2.30 *$ & $5.26 * *$ & $-25.09 * *$ & 0.16 & 1.90 & $-17.90 * *$ & 12.90 & -5.41 & $-16.67 * *$ \\
\hline 27. & CMS-1409A x RSSV-453 & 1.03 & $3.68 * *$ & $-26.22 * *$ & -0.63 & 0.95 & $-18.67 * *$ & $19.35 * *$ & 0.00 & -11.90 \\
\hline 28. & CMS-1409A x RSSV-313 & $-6.54 * *$ & $5.26 * *$ & $-25.09 * *$ & $-5.31 * *$ & 1.90 & $-17.90 * *$ & 9.68 & -8.11 & $-19.05 * *$ \\
\hline 29. & CMS-1409A x RSSV-512 & -1.25 & $3.68 * *$ & $-26.22 * *$ & -0.77 & 2.22 & $-17.65 * *$ & $34.38 * *$ & 10.26 & 2.38 \\
\hline \multirow[t]{4}{*}{30.} & CMS-1409A x RSSV-503 & 0.25 & $4.21 * *$ & $-25.84 * *$ & 0.16 & $2.54 *$ & $-17.39 * *$ & $33.33 * *$ & 10.53 & 0.00 \\
\hline & S.E. $($ Sij) \pm & 0.6379 & 0.7366 & 0.7366 & 1.1441 & 1.3211 & 1.3211 & 0.7231 & 0.8350 & 0.8350 \\
\hline & C.D. 5\% & 1.2768 & 1.4744 & 1.4744 & 2.2902 & 2.6445 & 2.6445 & 1.4475 & 1.6714 & 1.6714 \\
\hline & C.D. $1 \%$ & 1.6989 & 1.9617 & 1.9617 & 3.0472 & 3.5186 & 3.5186 & 1.9259 & 2.2238 & 2.2238 \\
\hline
\end{tabular}

Note: * Significant 4 at $5 \%$ level of significance, $* *$ Significant at $1 \%$ level of significance 
Table. 3 contd...

\begin{tabular}{|c|c|c|c|c|c|c|c|c|c|c|}
\hline \multirow{3}{*}{$\begin{array}{l}\text { Sr. } \\
\text { No. }\end{array}$} & \multirow[t]{3}{*}{ Crosses } & \multirow{2}{*}{\multicolumn{3}{|c|}{ Plant height (cm) }} & \multirow{2}{*}{\multicolumn{3}{|c|}{ Stem girth $(\mathrm{cm})$}} & \multicolumn{3}{|c|}{ Total biomass yield (t/ha) } \\
\hline & & & & & \multicolumn{2}{|c|}{5} & & \multicolumn{3}{|c|}{6} \\
\hline & & MP (H1) & BP(H2) & Check (H3) & MP (H1) & BP(H2) & Check (H3) & MP (H1) & BP (H2) & Check (H3) \\
\hline 1 & CMS-185A x RSSV-542 & $12.89 * *$ & $-9.71 * *$ & $-12.66 * *$ & -1.11 & -1.29 & $-31.17 * *$ & $34.46 * *$ & $14.76 * *$ & $-5.86 * *$ \\
\hline 2 & CMS-185A x RSSV-260 & $32.04 * *$ & $5.24 * *$ & $2.77 * *$ & $52.68 * *$ & $49.15 * *$ & $9.06 * *$ & $55.82 * *$ & $36.60 * *$ & $5.15 * *$ \\
\hline 3 & CMS-185A x RSSV-509 & $43.24 * *$ & $20.69 * *$ & $2.18 *$ & $59.74 * *$ & $43.29 * *$ & -0.08 & $47.42 * *$ & $27.14 * *$ & $1.71 *$ \\
\hline 4 & CMS-185A x RSSV-386 & $35.27 * *$ & $10.18 * *$ & 1.59 & $45.02 * *$ & $37.63 * *$ & $-4.02 * *$ & $43.38 * *$ & $22.25 * *$ & 0.51 \\
\hline 5 & CMS-185A x RSSV-498 & $28.52 * *$ & $2.49 *$ & -0.08 & $29.21 * *$ & $20.05 * *$ & $-16.28 * *$ & $32.18 * *$ & $15.79 * *$ & $-10.71 * *$ \\
\hline 6 & CMS-185A x RSSV-417 & $17.81 * *$ & $-2.82 * *$ & $-13.24 * *$ & $8.69 * *$ & $3.50 *$ & $-27.82 * *$ & $33.36 * *$ & $14.38 * *$ & $-7.27 * *$ \\
\hline 7 & CMS-185A x RSSV-453 & $17.64 * *$ & $-4.34 * *$ & $-11.40 * *$ & -2.12 & $-3.07 *$ & $-32.41 * *$ & $31.22 * *$ & $12.73 * *$ & $-8.96 * *$ \\
\hline 8 & CMS-185A x RSSV-313 & $32.92 * *$ & $8.27 * *$ & -0.17 & $-4.82 * *$ & $-5.23 * *$ & $-33.91 * *$ & $20.77 * *$ & -1.40 & $-9.66 * *$ \\
\hline 9 & CMS-185A x RSSV-512 & $31.66 * *$ & $5.89 * *$ & 0.92 & $31.86 * *$ & $25.88 * *$ & $-3.46 * *$ & $41.68 * *$ & $17.15 * *$ & $3.92 * *$ \\
\hline 10 & CMS-185A x RSSV-503 & $21.13 * *$ & -0.56 & $-10.14 * *$ & $-2.58 *$ & $-2.94 *$ & $-31.80 * *$ & $36.02 * *$ & $16.51 * *$ & $-5.26 * *$ \\
\hline 11 & ICMS-479A x RSSV-542 & $15.47 * *$ & $-10.75 * *$ & $-13.66 * *$ & $16.21 * *$ & $12.72 * *$ & $-21.69 * *$ & $33.02 * *$ & $13.77 * *$ & $-6.68 * *$ \\
\hline 12 & ICMS-479A x RSSV-260 & $38.94 * *$ & $7.04 * *$ & $4.53 * *$ & $58.39 * *$ & $49.92 * *$ & $9.62 * *$ & $63.02 * *$ & $43.23 * *$ & $10.25 * *$ \\
\hline 13 & ICMS-479A x RSSV-509 & $47.44 * *$ & $19.70 * *$ & 1.34 & $42.99 * *$ & $32.12 * *$ & $-13.72 * *$ & $47.95 * *$ & $27.87 * *$ & $2.29 * *$ \\
\hline 14 & ICMS-479A x RSSV-386 & $16.76 * *$ & $-8.18 * *$ & $-15.34 * *$ & -1.15 & $-3.17 *$ & $-36.77 * *$ & $24.29 * *$ & $6.20 * *$ & $-12.68 * *$ \\
\hline 15 & ICMS-479A x RSSV-498 & $12.99 * *$ & $-12.90 * *$ & $-15.09 * *$ & $10.24 * *$ & $5.64 * *$ & $-31.02 * *$ & $28.85 * *$ & $13.12 * *$ & $-12.76 * *$ \\
\hline 16. & ICMS-479A x RSSV-417 & $25.90 * *$ & 0.19 & $-10.56 * *$ & $-5.24 * *$ & $-6.85 * *$ & $-39.17 * *$ & $21.78 * *$ & $4.67 * *$ & $-15.14 * *$ \\
\hline 17. & ICMS-479A x RSSV-453 & $19.08 * *$ & $-6.52 * *$ & $-13.41 * *$ & $36.00 * *$ & $32.93 * *$ & $-9.10 * *$ & $21.91 * *$ & $4.95 * *$ & $-15.25 * *$ \\
\hline 18. & ICMS-479A x RSSV-313 & $21.16 * *$ & $-4.73 * *$ & $-12.15 * *$ & $14.49 * *$ & $11.31 * *$ & $-23.05 * *$ & $10.31 * *$ & $-9.75 * *$ & $-17.32 * *$ \\
\hline 19. & ICMS-479A x RSSV-512 & $21.68 * *$ & $-5.45 * *$ & $-9.89 * *$ & -2.14 & $-9.41 * *$ & $-30.53 * *$ & $22.48 * *$ & 1.48 & $-9.98 * *$ \\
\hline 20. & ICMS-479A x RSSV-503 & $31.15 * *$ & $3.90 * *$ & $-6.12 * *$ & $40.93 * *$ & $35.96 * *$ & $-4.47 * *$ & $9.70 * *$ & $-5.83 * *$ & $-23.42 * *$ \\
\hline 21. & CMS-1409A x RSSV-542 & $34.33 * *$ & 1.04 & $-2.26 *$ & $-17.80 * *$ & $-20.78 * *$ & $-44.96 * *$ & $20.67 * *$ & $9.39 * *$ & $-10.27 * *$ \\
\hline 22. & CMS-1409A x RSSV-260 & $38.07 * *$ & $3.52 * *$ & 1.09 & $54.91 * *$ & $45.71 * *$ & $6.54 * *$ & $46.37 * *$ & $36.59 * *$ & $5.14 * *$ \\
\hline 23. & CMS-1409A x RSSV-509 & $48.24 * *$ & $16.83 * *$ & -1.09 & $48.60 * *$ & $38.16 * *$ & $-10.98 * *$ & $11.03 * *$ & $1.79 *$ & $-18.57 * *$ \\
\hline 24. & CMS-1409A x RSSV-386 & $40.67 * *$ & $7.55 * *$ & -0.84 & $36.75 * *$ & $34.83 * *$ & $-13.12 * *$ & $30.54 * *$ & $18.22 * *$ & $-2.81 * *$ \\
\hline 25. & CMS-1409A x RSSV-498 & $19.43 * *$ & $-10.40 * *$ & $-12.66 * *$ & $27.53 * *$ & $22.99 * *$ & $-20.75 * *$ & $14.09 * *$ & $6.37 * *$ & $-17.96 * *$ \\
\hline 26. & CMS-1409A x RSSV-417 & $26.05 * *$ & $-2.54 *$ & $-12.99 * *$ & $25.59 * *$ & $24.27 * *$ & $-19.92 * *$ & $14.64 * *$ & $4.47 * *$ & $-15.30 * *$ \\
\hline 27. & CMS-1409A x RSSV-453 & $24.24 * *$ & $-5.16 * *$ & $-12.15 * *$ & $26.97 * *$ & $23.31 * *$ & $-15.68 * *$ & $29.28 * *$ & $18.02 * *$ & $-4.69 * *$ \\
\hline 28. & CMS-1409A x RSSV-313 & $29.01 * *$ & -1.36 & $-9.05 * *$ & $16.80 * *$ & $12.83 * *$ & $-21.99 * *$ & $17.65 * *$ & $1.64 *$ & $-6.88 * *$ \\
\hline 29. & CMS-1409A x RSSV-512 & $42.29 * *$ & $7.56 * *$ & $2.51 * *$ & $41.88 * *$ & $30.54 * *$ & 0.11 & $30.87 * *$ & $14.63 * *$ & $1.68 *$ \\
\hline \multirow[t]{4}{*}{30.} & CMS-1409A x RSSV-503 & $43.98 * *$ & $10.85 * *$ & 0.17 & $60.76 * *$ & $54.09 * *$ & $8.27 * *$ & $38.91 * *$ & $26.42 * *$ & $2.80 * *$ \\
\hline & S.E. $($ Sij $) \pm$ & 3.2090 & 3.7055 & 3.7055 & 0.0716 & 0.0826 & 0.0826 & 0.4527 & 0.5228 & 0.5228 \\
\hline & C.D. 5\% & 6.4235 & 7.4173 & 7.4173 & 0.1432 & 0.1654 & 0.1654 & 0.9062 & 1.0464 & 1.0464 \\
\hline & C.D. $1 \%$ & 8.5466 & 9.8687 & 9.8687 & 0.1906 & 0.2201 & 0.2201 & 1.2058 & 1.3923 & 1.3923 \\
\hline
\end{tabular}

Note: * Significant at $5 \%$ level of significance, $* *$ Significant at $1 \%$ level of significance 
Table. 3 contd...

\begin{tabular}{|c|c|c|c|c|c|c|c|c|c|c|}
\hline \multirow{3}{*}{$\begin{array}{l}\text { Sr. } \\
\text { No. }\end{array}$} & \multirow[t]{3}{*}{ Crosses } & \multicolumn{3}{|c|}{ Cane weight (t/ha) } & \multicolumn{3}{|c|}{ Cane harvest index (\%) } & \multicolumn{3}{|c|}{ Juice yield (lit/ha) } \\
\hline & & \multicolumn{3}{|c|}{7} & \multicolumn{3}{|c|}{8} & \multicolumn{3}{|c|}{9} \\
\hline & & MP (H1) & BP(H2) & Check (H3) & MP (H1) & BP(H2) & Check (H3) & MP (H1) & BP (H2) & Check (H3) \\
\hline 1 & CMS-185A x RSSV-542 & $57.29 * *$ & $22.50 * *$ & $-8.08 * *$ & $19.34 * *$ & $6.73 * *$ & $-2.36 * *$ & $-6.37 *$ & $-7.28 *$ & $-46.37 * *$ \\
\hline 2 & CMS-185A x RSSV-260 & $95.18 * *$ & $57.62 * *$ & $7.22 * *$ & $27.04 * *$ & $15.38 * *$ & $1.96 * *$ & $67.76 * *$ & $58.94 * *$ & 0.76 \\
\hline 3 & CMS-185A x RSSV-509 & $79.63 * *$ & $41.85 * *$ & $2.44 *$ & $24.01 * *$ & $11.56 * *$ & 0.72 & $68.12 * *$ & $59.11 * *$ & 1.09 \\
\hline 4 & CMS-185A x RSSV-386 & $72.06 * *$ & $33.83 * *$ & 0.77 & $22.46 * *$ & $9.47 * *$ & 0.26 & $43.91 * *$ & $39.77 * *$ & $-15.88 * *$ \\
\hline 5 & CMS-185A x RSSV-498 & $54.81 * *$ & $24.88 * *$ & $-14.81 * *$ & $18.80 * *$ & $7.85 * *$ & $-4.60 * *$ & $6.17 *$ & -0.34 & $-35.57 * *$ \\
\hline 6 & CMS-185A x RSSV-417 & $55.71 * *$ & $22.05 * *$ & $-10.04 * *$ & $18.99 * *$ & $6.71 * *$ & $-2.98 * *$ & $13.53 * *$ & $11.33 * *$ & $-34.31 * *$ \\
\hline 7 & CMS-185A x RSSV-453 & $52.22 * *$ & $19.57 * *$ & $-12.39 * *$ & $18.15 * *$ & $6.06 * *$ & $-3.77 * *$ & $-11.93 * *$ & $-23.04 * *$ & $-41.61 * *$ \\
\hline 8 & CMS-185A x RSSV-313 & $33.10 * *$ & -1.94 & $-13.35 * *$ & $13.78 * *$ & -0.55 & $-4.09 * *$ & -1.98 & -5.01 & $-42.57 * *$ \\
\hline 9 & CMS-185A x RSSV-512 & $67.27 * *$ & $25.14 * *$ & $5.50 * *$ & $21.45 * *$ & $6.82 * *$ & $1.53 * *$ & $62.42 * *$ & $50.20 * *$ & 0.29 \\
\hline 10 & CMS-185A x RSSV-503 & $60.08 * *$ & $25.27 * *$ & $-7.24 * *$ & $19.98 * *$ & $7.52 * *$ & $-2.09 * *$ & $-9.96 * *$ & $-25.42 * *$ & $-35.57 * *$ \\
\hline 11 & ICMS-479A x RSSV-542 & $54.81 * *$ & $20.99 * *$ & $-9.21 * *$ & $18.66 * *$ & $6.35 * *$ & $-2.71 * *$ & $19.00 * *$ & $10.77 * *$ & $-35.92 * *$ \\
\hline 12 & ICMS-479A x RSSV-260 & $107.31 * *$ & $68.04 * *$ & $14.30 * *$ & $28.89 * *$ & $17.30 * *$ & $3.66 * *$ & $93.25 * *$ & $72.60 * *$ & $9.41 * *$ \\
\hline 13 & ICMS-479A x RSSV-509 & $80.39 * *$ & $42.96 * *$ & $3.25 * *$ & $24.02 * *$ & $11.80 * *$ & $0.93 *$ & $72.81 * *$ & $54.19 * *$ & -2.04 \\
\hline 14 & ICMS-479A x RSSV-386 & $40.28 * *$ & $9.49 * *$ & $-17.55 * *$ & $15.09 * *$ & $3.09 * *$ & $-5.58 * *$ & 4.28 & -4.69 & $-42.63 * *$ \\
\hline 15 & ICMS-479A x RSSV-498 & $49.06 * *$ & $20.69 * *$ & $-17.66 * *$ & $17.28 * *$ & $6.69 * *$ & $-5.62 * *$ & $-6.93 * *$ & $-17.58 * *$ & $-46.72 * *$ \\
\hline 16. & ICMS-479A x RSSV-417 & $36.18 * *$ & $7.12 * *$ & $-21.05 * *$ & $13.88 * *$ & $2.34 * *$ & $-6.96 * *$ & $-5.74 *$ & $-13.07 * *$ & $-48.70 * *$ \\
\hline 17. & ICMS-479A x RSSV-453 & $36.44 * *$ & $7.55 * *$ & $-21.19 * *$ & $13.93 * *$ & $2.48 * *$ & $-7.02 * *$ & $-16.62 * *$ & $-30.92 * *$ & $-47.59 * *$ \\
\hline 18. & ICMS-479A x RSSV-313 & $16.28 * *$ & $-14.06 * *$ & $-24.06 * *$ & $8.73 * *$ & $-4.78 * *$ & $-8.16 * *$ & $26.64 * *$ & $15.52 * *$ & $-30.16 * *$ \\
\hline 19. & ICMS-479A x RSSV-512 & $36.10 * *$ & 2.15 & $-13.88 * *$ & $14.21 * *$ & 0.66 & $-4.33 * *$ & $10.93 * *$ & -3.13 & $-35.32 * *$ \\
\hline 20. & ICMS-479A x RSSV-503 & $16.00 * *$ & $-8.91 * *$ & $-32.55 * *$ & $7.71 * *$ & $-3.27 * *$ & $-11.92 * *$ & $-34.32 * *$ & $-48.21 * *$ & $-55.26 * *$ \\
\hline 21. & CMS-1409A x RSSV-542 & $33.13 * *$ & $14.23 * *$ & $-14.28 * *$ & $11.06 * *$ & $4.43 * *$ & $-4.46 * *$ & $54.33 * *$ & $42.01 * *$ & $-17.85 * *$ \\
\hline 22. & CMS-1409A x RSSV-260 & $75.98 * *$ & $57.49 * *$ & $7.13 * *$ & $20.63 * *$ & $15.30 * *$ & $1.89 * *$ & $86.73 * *$ & $64.96 * *$ & $4.57 * *$ \\
\hline 23. & CMS-1409A x RSSV-509 & $17.82 * *$ & 2.74 & $-25.80 * *$ & $6.66 * *$ & 0.93 & $-8.88 * *$ & $29.10 * *$ & $13.93 * *$ & $-27.61 * *$ \\
\hline 24. & CMS-1409A x RSSV-386 & $48.94 * *$ & $27.61 * *$ & $-3.91 * *$ & $14.85 * *$ & $7.94 * *$ & $-1.14 *$ & $51.72 * *$ & $37.13 * *$ & $-17.47 * *$ \\
\hline 25. & CMS-1409A x RSSV-498 & $23.06 * *$ & $9.99 * *$ & $-24.96 * *$ & $8.22 * *$ & $3.39 * *$ & $-8.54 * *$ & 0.76 & $-11.74 * *$ & $-42.94 * *$ \\
\hline 26. & CMS-1409A x RSSV-417 & $23.56 * *$ & $6.82 * *$ & $-21.27 * *$ & $8.42 * *$ & $2.25 * *$ & $-7.04 * *$ & $24.80 * *$ & $13.81 * *$ & $-32.84 * *$ \\
\hline 27. & CMS-1409A x RSSV-453 & $47.19 * *$ & $27.56 * *$ & $-6.53 * *$ & $14.51 * *$ & $8.09 * *$ & $-1.93 * *$ & -0.75 & $-18.58 * *$ & $-38.23 * *$ \\
\hline 28. & CMS-1409A x RSSV-313 & $27.29 * *$ & 2.34 & $-9.57 * *$ & $9.73 * *$ & 0.70 & $-2.88 * *$ & $14.06 * *$ & 2.88 & $-37.80 * *$ \\
\hline 29. & CMS-1409A x RSSV-512 & $48.36 * *$ & $21.45 * *$ & $2.39 *$ & $14.69 * *$ & $5.95 * *$ & 0.70 & $78.16 * *$ & $53.93 * *$ & 2.78 \\
\hline 30. & CMS-1409A x RSSV-503 & $62.70 * *$ & $40.38 * *$ & $3.95 * *$ & $17.84 * *$ & $11.05 * *$ & $1.12 *$ & $46.19 * *$ & $14.23 * *$ & -1.32 \\
\hline & S.E. (Sij) \pm & 0.4755 & 0.5490 & 0.5490 & 0.2871 & 0.3315 & 0.3315 & 194.2779 & 224.3328 & 224.3328 \\
\hline & C.D. $5 \%$ & 0.9518 & 1.0990 & 1.0990 & 0.5747 & 0.6636 & 0.6636 & 388.8 & 449.0495 & 449.0495 \\
\hline & C.D. $1 \%$ & 1.2663 & 1.4622 & 1.4622 & 0.7647 & 0.8830 & 0.8830 & 883 & 597.4657 & 597.4657 \\
\hline
\end{tabular}

Note: * Significant at $5 \%$ level of significance, $* *$ Significant at $1 \%$ level of significance 
Table. 3 contd...

\begin{tabular}{|c|c|c|c|c|c|c|c|c|c|c|}
\hline \multirow{3}{*}{$\begin{array}{l}\text { Sr. } \\
\text { No. }\end{array}$} & \multirow[t]{3}{*}{ Crosses } & \multicolumn{3}{|c|}{ Brix (\%) } & \multicolumn{3}{|c|}{ Reducing sugar (\%) } & \multicolumn{3}{|c|}{ Total sugar (\%) } \\
\hline & & \multicolumn{3}{|c|}{10} & \multicolumn{3}{|c|}{11} & \multicolumn{3}{|c|}{12} \\
\hline & & MP (H1) & $\mathbf{B P}(\mathbf{H} 2)$ & Check (H3) & MP (H1) & BP(H2) & Check (H3) & MP (H1) & BP (H2) & Check (H3) \\
\hline 1 & CMS-185A x RSSV-542 & 0.90 & -3.45 & 1.82 & $20.83 * *$ & $34.88 * *$ & $-17.14 * *$ & -0.69 & $-23.47 * *$ & $-18.47 * *$ \\
\hline 2 & CMS-185A x RSSV-260 & -0.97 & -3.77 & $-7.27 * *$ & $-8.26 *$ & $16.28 * *$ & $-28.57 * *$ & $41.32 * *$ & $18.79 * *$ & 0.57 \\
\hline 3 & CMS-185A x RSSV-509 & -3.77 & -3.77 & $-7.27 * *$ & $43.82 * *$ & $48.84 * *$ & $-8.57 *$ & $29.39 * *$ & $5.61 * *$ & $-3.69 *$ \\
\hline 4 & CMS-185A x RSSV-386 & 0.00 & -1.89 & $-5.45 *$ & 2.65 & $34.88 * *$ & $-17.14 * *$ & $46.27 * *$ & $28.95 * *$ & -2.56 \\
\hline 5 & CMS-185A x RSSV-498 & -3.70 & $-5.45 *$ & $-5.45 *$ & 4.35 & 11.63 & $-31.43 * *$ & $36.26 * *$ & $11.21 * *$ & 1.42 \\
\hline 6 & CMS-185A x RSSV-417 & -0.95 & -1.89 & $-5.45 *$ & $40.38 * *$ & $69.77 * *$ & 4.29 & $36.92 * *$ & $7.61 * *$ & $8.52 * *$ \\
\hline 7 & CMS-185A x RSSV-453 & $7.84 * *$ & 3.77 & 000 & 5.15 & $18.60 * *$ & $-27.14 * *$ & $15.07 * *$ & $-4.55 *$ & $-16.48 * *$ \\
\hline 8 & CMS-185A x RSSV-313 & $14.56 * *$ & $11.32 * *$ & $7.27 * *$ & 3.92 & $23.26 * *$ & $-24.29 * *$ & $20.83 * *$ & -1.85 & $-9.38 * *$ \\
\hline 9 & CMS-185A x RSSV-512 & 0.00 & -1.89 & $-5.45 *$ & $38.00 * *$ & $60.47 * *$ & -1.43 & $38.46 * *$ & $13.56 * *$ & 2.27 \\
\hline 10 & CMS-185A x RSSV-503 & -2.78 & -4.55 & -4.55 & -2.33 & -2.33 & $-40.00 * *$ & $35.36 * *$ & $20.93 * *$ & $-11.36 * *$ \\
\hline 11 & ICMS-479A x RSSV-542 & 4.59 & -1.72 & 3.64 & $64.71 * *$ & $118.75 * *$ & 0.00 & $-10.92 * *$ & $-30.40 * *$ & $-25.85 * *$ \\
\hline 12 & ICMS-479A x RSSV-260 & $16.83 * *$ & $15.69 * *$ & $7.27 * *$ & $40.82 * *$ & $115.62 * *$ & -1.43 & $41.45 * *$ & $20.81 * *$ & 2.27 \\
\hline 13 & ICMS-479A x RSSV-509 & $17.31 * *$ & $15.09 * *$ & $10.91 * *$ & $28.21 * *$ & $56.25 * *$ & $-28.57 * *$ & $32.71 * *$ & $9.97 * *$ & 0.28 \\
\hline 14 & ICMS-479A x RSSV-386 & 1.96 & 1.96 & $-5.45 *$ & $-27.45 * *$ & 15.62 & $-47.14 * *$ & 1.05 & $-9.40 * *$ & $-31.53 * *$ \\
\hline 15 & ICMS-479A x RSSV-498 & $5.66 *$ & 1.82 & 1.82 & $13.58 *$ & $43.75 * *$ & $-34.29 * *$ & $21.80 * *$ & 0.93 & $-7.95 * *$ \\
\hline 16. & ICMS-479A x RSSV-417 & -4.85 & $-5.77 *$ & $-10.91 * *$ & $11.83 *$ & $62.50 * *$ & $-25.71 * *$ & $8.13 * *$ & $-13.80 * *$ & $-13.07 * *$ \\
\hline 17. & ICMS-479A x RSSV-453 & 2.00 & 0.00 & $-7.27 * *$ & $41.86 * *$ & $90.62 * *$ & $-12.86 * *$ & $30.64 * *$ & $10.06 * *$ & $-3.69 *$ \\
\hline 18. & ICMS-479A x RSSV-313 & 2.97 & 1.96 & $-5.45 *$ & $14.29 * *$ & $62.50 * *$ & $-25.71 * *$ & -3.73 & $-20.62 * *$ & $-26.70 * *$ \\
\hline 19. & ICMS-479A x RSSV-512 & 1.96 & 1.96 & $-5.45 *$ & $12.36 *$ & $56.25 * *$ & $-28.57 * *$ & $15.15 * *$ & $-4.10 *$ & $-13.64 * *$ \\
\hline 20. & ICMS-479A x RSSV-503 & $5.66 *$ & 1.82 & 1.82 & $33.33 * *$ & $56.25 * *$ & $-28.57 * *$ & $43.28 * *$ & $30.23 * *$ & $-4.55 *$ \\
\hline 21. & CMS-1409A x RSSV-542 & -3.64 & $-8.62 * *$ & -3.64 & $10.84 *$ & $53.33 * *$ & $-34.29 * *$ & $19.05 * *$ & $-6.67 * *$ & -0.57 \\
\hline 22. & CMS-1409A x RSSV-260 & 1.96 & 0.00 & $-5.45 *$ & $60.42 * *$ & $156.67 * *$ & $10.00 * *$ & $39.73 * *$ & $19.80 * *$ & 1.42 \\
\hline 23. & CMS-1409A x RSSV-509 & $21.90 * *$ & $20.75 * *$ & $16.36 * *$ & $21.05 * *$ & $53.33 * *$ & $-34.29 * *$ & $40.82 * *$ & $17.13 * *$ & $6.82 * *$ \\
\hline 24. & CMS-1409A x RSSV-386 & $14.56 * *$ & $13.46 * *$ & $7.27 * *$ & $48.00 * *$ & $146.67 * *$ & 5.71 & $58.66 * *$ & $42.86 * *$ & $7.95 * *$ \\
\hline 25. & CMS-1409A x RSSV-498 & $14.02 * *$ & $10.91 * *$ & $10.91 * *$ & 3.80 & $36.67 * *$ & $-41.43 * *$ & $32.96 * *$ & $10.59 * *$ & 0.85 \\
\hline 26. & CMS-1409A x RSSV-417 & $7.69 * *$ & $7.69 * *$ & 1.82 & $62.64 * *$ & $146.67 * *$ & 5.71 & $33.80 * *$ & $7.04 * *$ & $7.95 * *$ \\
\hline 27. & CMS-1409A x RSSV-453 & $-6.93 * *$ & $-9.62 * *$ & $-14.55 * *$ & -4.76 & $33.33 * *$ & $-42.86 * *$ & $27.83 * *$ & $8.12 * *$ & $-5.40 * *$ \\
\hline 28. & CMS-1409A x RSSV-313 & $11.76 * *$ & $9.62 * *$ & 3.64 & $73.03 * *$ & $156.67 * *$ & $10.00 * *$ & $31.97 * *$ & $9.23 * *$ & 0.85 \\
\hline 29. & CMS-1409A x RSSV-512 & $12.62 * *$ & $11.54 * *$ & $5.45 *$ & $49.43 * *$ & $116.67 * *$ & -7.14 & $35.47 * *$ & $13.25 * *$ & 1.99 \\
\hline 30. & CMS-1409A x RSSV-503 & $10.28 * *$ & $7.27 * *$ & $7.27 * *$ & $47.95 * *$ & $80.00 * *$ & $-22.86 * *$ & $51.17 * *$ & $37.98 * *$ & 1.14 \\
\hline & S.E. $($ Sij $) \pm$ & 0.4253 & 0.4911 & 0.4911 & 0.0748 & 0.0863 & 0.0863 & 0.1827 & 0.2109 & 0.2109 \\
\hline & C.D. 5\% & 0.8513 & 0.9830 & 0.9830 & 0.1496 & 0.1728 & 0.1728 & 0.3657 & 0.4222 & 0.4222 \\
\hline & C.D. $1 \%$ & 1.1327 & 1.3079 & 1.3079 & 0.1991 & 0.2299 & 0.2299 & 0.4865 & 0.5618 & 0.5618 \\
\hline
\end{tabular}

Note: * Significant at $5 \%$ level of significance, $* *$ Significant at $1 \%$ level of significance 
Table. 3 contd...

\begin{tabular}{|c|c|c|c|c|c|c|c|}
\hline \multirow{3}{*}{$\begin{array}{l}\text { Sr. } \\
\text { No. }\end{array}$} & \multirow[t]{3}{*}{ Crosses } & \multicolumn{3}{|c|}{ Non reducing sugar $(\%)$} & \multicolumn{3}{|c|}{ Ethanol (lit/ha) } \\
\hline & & \multicolumn{3}{|c|}{13} & \multicolumn{3}{|c|}{14} \\
\hline & & MP (H1) & BP(H2) & Check (H3) & MP (H1) & BP(H2) & Check (H3) \\
\hline 1 & CMS-185A x RSSV-542 & $-5.05 *$ & $-29.01 * *$ & $-18.94 * *$ & $-7.12 *$ & $-28.84 * *$ & $-56.21 * *$ \\
\hline 2 & CMS-185A x RSSV-260 & $55.52 * *$ & $31.40 * *$ & $7.73 * *$ & $135.38 * *$ & $89.80 * *$ & 1.50 \\
\hline 3 & CMS-185A x RSSV-509 & $26.90 * *$ & 0.25 & -2.23 & $115.18 * *$ & $68.51 * *$ & -2.49 \\
\hline 4 & CMS-185A x RSSV-386 & $61.70 * *$ & $47.32 * *$ & 1.35 & $110.12 * *$ & $80.99 * *$ & $-17.94 * *$ \\
\hline 5 & CMS-185A x RSSV-498 & $43.63 * *$ & $14.10 * *$ & $9.61 * *$ & $42.59 * *$ & $10.93 * *$ & $-34.62 * *$ \\
\hline 6 & CMS-185A x RSSV-417 & $36.08 * *$ & $4.97 *$ & $9.40 * *$ & $54.35 * *$ & $19.65 * *$ & $-28.77 * *$ \\
\hline 7 & CMS-185A x RSSV-453 & $17.07 * *$ & -4.65 & $-14.26 * *$ & -1.92 & $-26.71 * *$ & $-51.42 * *$ \\
\hline 8 & CMS-185A x RSSV-313 & $25.09 * *$ & 0.19 & $-5.85 *$ & $17.18 * *$ & $-7.01 *$ & $-48.10 * *$ \\
\hline 9 & CMS-185A x RSSV-512 & $38.23 * *$ & $11.55 * *$ & 2.77 & $120.49 * *$ & $70.26 * *$ & 2.49 \\
\hline 10 & CMS-185A x RSSV-503 & $44.34 * *$ & $25.73 * *$ & -4.18 & $18.75 * *$ & $-9.92 * *$ & $-42.92 * *$ \\
\hline 11 & ICMS-479A x RSSV-542 & $-23.75 * *$ & $-40.87 * *$ & $-32.48 * *$ & 4.03 & $-22.86 * *$ & $-52.53 * *$ \\
\hline 12 & ICMS-479A x RSSV-260 & $42.09 * *$ & $25.56 * *$ & 2.94 & $169.07 * *$ & $109.31 * *$ & $11.94 * *$ \\
\hline 13 & ICMS-479A x RSSV-509 & $33.91 * *$ & $10.15 * *$ & $7.41 * *$ & $124.01 * *$ & $69.54 * *$ & -1.90 \\
\hline 14 & ICMS-479A x RSSV-386 & $9.32 * *$ & 4.64 & $-28.01 * *$ & 4.48 & $-13.51 * *$ & $-60.79 * *$ \\
\hline 15 & ICMS-479A x RSSV-498 & $24.16 * *$ & 2.73 & -1.31 & $10.57 * *$ & $-16.83 * *$ & $-50.99 * *$ \\
\hline 16. & ICMS-479A x RSSV-417 & $7.62 * *$ & $-13.71 * *$ & $-10.07 * *$ & 0.09 & $-24.97 * *$ & $-55.34 * *$ \\
\hline 17. & ICMS-479A x RSSV-453 & $29.10 * *$ & $9.70 * *$ & -1.35 & 5.39 & $-23.67 * *$ & $-49.41 * *$ \\
\hline 18. & ICMS-479A x RSSV-313 & $-7.69 * *$ & $-22.94 * *$ & $-27.59 * *$ & $19.13 * *$ & $-8.71 * *$ & $-49.05 * *$ \\
\hline 19. & ICMS-479A x RSSV-512 & $15.74 * *$ & -2.62 & $-10.28 * *$ & $23.87 * *$ & $-7.49 *$ & $-44.31 * *$ \\
\hline 20. & ICMS-479A x RSSV-503 & $45.91 * *$ & $33.18 * *$ & 1.49 & $-8.03 *$ & $-32.44 * *$ & $-57.19 * *$ \\
\hline 21. & CMS-1409A x RSSV-542 & $20.61 * *$ & $-5.59 *$ & $7.80 * *$ & $79.75 * *$ & $32.82 * *$ & $-18.26 * *$ \\
\hline 22. & CMS-1409A x RSSV-260 & $35.83 * *$ & $21.41 * *$ & -0.46 & $156.18 * *$ & $98.52 * *$ & $6.17 * *$ \\
\hline 23. & CMS-1409A x RSSV-509 & $44.74 * *$ & $20.29 * *$ & $17.30 * *$ & $77.54 * *$ & $33.88 * *$ & $-22.53 * *$ \\
\hline 24. & CMS-1409A x RSSV-386 & $62.83 * *$ & $57.84 * *$ & $8.58 * *$ & $138.50 * *$ & $96.60 * *$ & $-10.87 * *$ \\
\hline 25. & CMS-1409A x RSSV-498 & $38.41 * *$ & $15.73 * *$ & $11.17 * *$ & $30.29 * *$ & -2.35 & $-42.45 * *$ \\
\hline 26. & CMS-1409A x RSSV-417 & $28.74 * *$ & 4.25 & $8.65 * *$ & $63.38 * *$ & $22.05 * *$ & $-27.35 * *$ \\
\hline 27. & CMS-1409A x RSSV-453 & $34.08 * *$ & $15.18 * *$ & 3.58 & $21.85 * *$ & $-12.05 * *$ & $-41.70 * *$ \\
\hline 28. & CMS-1409A x RSSV-313 & $24.54 * *$ & 5.06 & -1.28 & $47.31 * *$ & $12.46 * *$ & $-37.23 * *$ \\
\hline 29. & CMS-1409A x RSSV-512 & $32.93 * *$ & $13.05 * *$ & 4.15 & $134.05 * *$ & $74.20 * *$ & $4.86 * *$ \\
\hline 30. & CMS-1409A x RSSV-503 & $52.09 * *$ & $40.48 * *$ & $7.06 * *$ & $114.83 * *$ & $57.27 * *$ & -0.36 \\
\hline & S.E. $($ Sij) \pm & 0.2009 & 0.2320 & 0.2320 & 13.3060 & 15.3644 & 15.3644 \\
\hline & C.D. $5 \%$ & 0.4021 & 0.4643 & 0.4643 & 26.6348 & 30.7552 & 30.7552 \\
\hline & C.D. $1 \%$ & 0.5350 & 0.6178 & 0.6178 & 35.4379 & 40.9201 & 40.9201 \\
\hline
\end{tabular}

Note: * Significant at $5 \%$ level of significance, ${ }^{* *}$ Significant at $1 \%$ level of significance 
The cross by ICMS-479 x RSSV-386 ($47.14 \%)$ exhibited significant negative standard heterosis over check Phule Vasundhara, while the cross CMS-1409 $\mathrm{x}$ RSSV-260 (10\%) showed maximum significant positive standard heterosis over Phule Vasundhara.

Range of standard heterosis from -2.56 to 8.52 per cent for percentage of total sugar over Phule Vasundhara was observed. Out of 30 crosses 4 crosses exhibited positive and significant standard heterosis for this trait. The cross CMS-185 x RSSV-417 (8.52\%) exhibited maximum positive standard heterosis followed by cross CMS-1409 $\mathrm{x}$ RSSV-386 and -1409 x RSSV- (7.95), Average heterosis for percentage of total sugar ranged from -5.05 to 62.83 per cent. The heterobeltiosis ranged from -2.62 to 57.84 per cent. 16 crosses recorded significant positive heterobeltiosis. Maximum positive heterobeltiosis observed in cross CMS-1409 x RSSV-386 (57.84\%) followed by CMS-185 x RSSV-386 (47.32\%). Range of standard heterosis from -0.46 to 17.30 per cent for percentage of non reducing sugar over Phule Vasundhara was observed. Out of 30 crosses 10 crosses exhibited positive and significant standard heterosis for this trait. The cross CMS-1409 x RSSV-509 (17.30\%) exhibited maximum positive standard heterosis followed by cross CMS-1409 x RSSV-498 (1.17). For ethanol yield, heterosis over mid parent ranged from -1.92 per cent (CMS-185 x RSSV-453) to 169.07 per cent (ICMS-479 $\mathrm{X}$ RSSV-260). As many as 23 out of 30 hybrids recorded positive significant heterosis over mid parent. The heterosis over better parent was ranged from -7.01 per cent (CMS$185 \times$ RSSV-313) to 109.31 per cent (ICMS479 X RSSV-260). A total of 16 hybrids recorded positive significant heterosis over better parent. The cross ICMS-479 X RSSV$260(109.31 \%)$ recorded highest positive significant heterosis over better parent.
Standard heterosis over check hybrid Phule Vasundhara ranged from -0.36 per cent (CMS-1409 x RSSV-503) to 28.77 per cent (CMS-185 X RSSV-417). A total of 4 hybrids recorded positive significant heterosis over Phule Vasundhara.

The highest significant standard heterosis recorded in the cross, CMS-185 x RSSV-417 (28.77\%), followed by cross ICMS-479 x RSSV-260 (11.94\%) and CMS-1409 x RSSV260 (43.61\% (Table 3).

In conclusion, the heterosis studies indicated the expression of relative heterosis, heterobeltosis and standard heterosis in several crosses for most of the characters in both desirable direction as well as undesirable direction. At least 7 to 10 crosses were shown significant positive heterosis over standard check Phule Vasundhara for the traits plant height, stalk yield, brix (\%), total biomass yield, cane yield, total sugar and ethanol yield but for juice yield only two crosses viz. ICMS-479A x RSSV-260, CMS-1409A x RSSV-260 were shown significant positive heterosis over standard check Phule Vasundhara. The top five crosses that showed significant heterosis over standard check Phule Vasundhara for the traits plant height, stem thickness, biomass yield and ethanol yield were viz., ICMS-479 x RSSV-260, ICMS-479 x RSSV-260, CMS-185 x RSSV260, CMS-1409A x RSSV-512, CMS-185A x RSSV-512. Also these crosses were shown significant positive sca effects for majority of the traits studied.

\section{Acknowledgement}

The Authors are sincerely grateful to Department of Agriculture Botany and All India Coordinated Sorghum Improvement Project, Mahatma Phule Krushi Vidyapeeth Rahuri, for providing necessary facilities for complete research work. 


\section{References}

Agrawal, M., Singh, R. and Shrotria, R.K. 2005. Combining ability analysis for forage yield and quality characters in sorghum [Sorghum bicolour (L.) Moench]. Forage Res., 31(1):8-11.

Bahadure, D.M., Marker, S., Umakanth, A.V., Prabhakar, Ramteke, P.W., Patil J.V. and Rana, B.S. 2015. Combining ability and heterosis on millable stalk and sugar concentration for bioethanol production across environments in sweet sorghum [Sorghum bicolour (L.) Moench.]. Electronic Journal of Plant Breeding, 6(1):58-65.

Fonseca, A. and Patterson. F.L. 1968. Hybrid vigour in a seven parent diallel cross in common winter wheat ( $T$. aestivum L.). Crop Sci., 8: 85-88.

Kumar, S., Srinivasa, I., Belum, R.P., Reddy, V.S., Ravindrababu, V. And Reddy, K.H.P. 2016. Heterosis and inbreeding depression in tropical sweet sorghum [Sorghum bicolour (L.) Moench]. Crop Res., 6(51):1.

Meshram, M.P., Atale, S.B., Murumkar, R.D. and Raut, P.B. 2005. Heterosis and heterobeltiosis studies in sweet sorghum. Ann. Plant Physiol., 19: 9698.

Pfeiffer, T.W., Bitzer, M.J., Toy, J.J. and Pedersen, J.F. 2010. Heterosis in sweet sorghum and selection of a new sweet sorghum hybrids for use in syrup production in Appalachia. Crop
Science, 50: 1788-1794. production. Agron. J., 46: 20-23.

National Convention of Sugar Production from Agriculture Products, 13-16 March, 1994, Shahid Chamran University, Ahwaz, Iran.

Rajashekhar, M.K. 2007. Studies on heterosis, combining ability, stability and molecular diversity in sweet sorghum [Sorghum bicolor (L.) Moench]. Karnataka J. Agric. Sci., 20: 889.

Sandeep, R.G., Gururaja Rao, M.R., Chikkalingaiah and Jagadeesh, B.N. 2009. Heterosis studies in sweet sorghum [Sorghum bicolor (L.) Moench]. Indian J. Crop Science, 4: 8791.

Sidramappa, T., Gururaja, R., Ramesh, S. and Kulkarni, R.S. 2012. Heterosis for ethanol yield and its attributing traits in sweet sorghum [Sorghum bicolor (L.) Moench]. International Journal of Plant Sciences, 7 (1):151-154.

Tariq, A.S., Akram, Z., Shabir, G., Khan, K.S. and Iqbal, M.S. 2014. Heterosis and Combining ability for quantitative traits in fodder sorghum. Electronical Journal of Plant Breeding, 3(2):775775.

Vinaykumar, R. 2009. Genetic analysis of bio-energy traits in sweet sorghum [Sorghum bicolor (L.) Moench]. M. Sc. (Agri.) thesis, University of Agricultural Sciences, Bangalore, 114.

\section{How to cite this article:}

Aru, S.R., D.V. Kusalkar, U.S. Dalvi, M.S. Shinde, A.S. Totre, A.S. Jadhav and Wani, V.S. 2020. Heterosis for Cane, Juice Yield and its Component Traits in Sweet Sorghum. Int.J.Curr.Microbiol.App.Sci. 9(09): 2730-2744. doi: https://doi.org/10.20546/ijcmas.2020.909.340 